\title{
Neurodevelopmental and neurobehavioral characteristics in males and females with CDKL5 duplications
}

\author{
Przemyslaw Szafranski ${ }^{1,6}$, Sailaja Golla ${ }^{2,6}$, Weihong Jin ${ }^{1}$, Ping Fang ${ }^{1}$, Patricia Hixson ${ }^{1}$, Reuben Matalon ${ }^{3}$, \\ Daniel Kinney ${ }^{4}$, Hans-georg Bock ${ }^{5}$, William Craigen ${ }^{1}$, Janice L Smith ${ }^{1}$, Weimin Bi ${ }^{1}$, Ankita Patel ${ }^{1}$, \\ Sau Wai Cheung ${ }^{1}$, Carlos A Bacino ${ }^{1}$ and Paweł Stankiewicz ${ }^{\star, 1}$
}

Point mutations and genomic deletions of the CDKL5 (STK9) gene on chromosome Xp22 have been reported in patients with severe neurodevelopmental abnormalities, including Rett-like disorders. To date, only larger-sized (8-21 Mb) duplications harboring CDKL5 have been described. We report seven females and four males from seven unrelated families with CDKL5 duplications $540-935 \mathrm{~kb}$ in size. Three families of different ethnicities had identical $667 \mathrm{~kb}$ duplications containing only the shorter CDKL5 isoform. Four affected boys, 8-14 years of age, and three affected girls, 6-8 years of age, manifested autistic behavior, developmental delay, language impairment, and hyperactivity. Of note, two boys and one girl had macrocephaly. Two carrier mothers of the affected boys reported a history of problems with learning and mathematics while at school. None of the patients had epilepsy. Similarly to CDKL5 mutations and deletions, the $\mathrm{X}$-inactivation pattern in all six studied females was random. We hypothesize that the increased dosage of CDKL5 might have affected interactions of this kinase with its substrates, leading to perturbation of synaptic plasticity and learning, and resulting in autistic behavior, developmental and speech delay, hyperactivity, and macrocephaly.

European Journal of Human Genetics (2015) 23, 915-921; doi:10.1038/ejhg.2014.217; published online 15 October 2014

\section{INTRODUCTION}

Rett syndrome (OMIM 312750) is an X-linked progressive neurodevelopmental disorder primarily affecting girls and characterized by apparently normal psychomotor development during the first 6-18 months of life, followed by a short period of developmental stagnation, rapid regression in language and motor skills, and longterm stability, as well as epilepsy in the majority of patients. Point mutations or genomic deletions in MECP2 have been identified in greater than $90 \%$ of patients with typical Rett syndrome. ${ }^{1,2}$ In $30-50 \%$ of patients with atypical Rett syndrome, ${ }^{3}$ negative for alterations in $M E C P 2$, intragenic changes in CDKL $5^{4-8}$ or in FOXG1 $1^{9-11}$ have been reported.

CDKL5 (OMIM 300203) encodes a proline-directed kinase belonging to the cyclin-dependent, mitogen-activated, glycogen synthase, and CDC-like family of serine/threonine kinases. It contains an N-terminal highly conserved catalytic domain and a large C-terminal region involved in localizing the protein to the cytoplasm through a mechanism depending on active nuclear export. CDKL5-signaling cascades have been proposed to be involved in synaptic plasticity and learning, affecting spines, dendritic branching, and actin cytoskeleton in the cytoplasm and neuronal activity-dependent gene expression in the nucleus. ${ }^{12,13}$

To date, over 200 sequence variants or genomic deletions in CDKL5 have been reported in both female and male patients with $\mathrm{X}$-linked dominant early-onset seizures manifesting before 5 months of age, severe intellectual disability with absent speech, and Rett-like features such as hand stereotypies and deceleration of head growth classified as Epileptic encephalopathy, early infantile, 2 (OMIM 300672) or Angelman syndrome-like (OMIM 105830). ${ }^{7,14-18}$ The phenotypic resemblance to Rett syndrome has been proposed to result from similar functions or interactions between CDKL5 and MECP2 in their molecular pathways during neurodevelopment (OMIM 30005); 4,13,19-23 however, more studies are needed to verify this hypothesis. Most recently, Livide et $a^{24}$ showed that a subunit of glutamate receptor ligand-gated ion channel, GluD1, encoded by GRID1, is a commonly altered player in neuronal differentiation from both MECP2-mutated and CDKL5-mutated iPS cells and suggested that it could be a new therapeutic target for Rett syndrome.

In contrast to phenotypically well-defined genomic duplications of MECP2 in males (OMIM 300260) and of FOXG1, ${ }^{25-30}$ the clinical consequences of increased dosage of CDKL5 are poorly understood. Thus far, only larger-sized (8-21 Mb) Xp22 duplications involving CDKL5 have been described, ${ }^{31-34}$ making phenotypic contribution of CDKL5 duplication challenging to dissect. Here, we correlate genotypes with phenotypes of four males and seven females from seven unrelated families with CDKL5 duplications smaller than $1 \mathrm{Mb}$ in size.

\footnotetext{
${ }^{1}$ Department of Molecular and Human Genetics, Baylor College of Medicine, Houston, TX, USA; ${ }^{2}$ Departments of Pediatrics and Neurology, University of Texas Southwestern Medical Center, Dallas, TX, USA; ${ }^{3}$ Division of General Academic Pediatrics, Department of Pediatrics, The University of Texas Medical Branch at Galveston, Galveston, TX, USA; ${ }^{4}$ Memorial Children's Hospital Navarre Pediatrics South Bend, South Bend, IN, USA; ${ }^{5}$ Department of Pediatrics, University of Mississippi Medical Center, Jackson, MS, USA *Correspondence: Dr P Stankiewicz, Department of Molecular and Human Genetics, Baylor College of Medicine, One Baylor Plaza, Rm ABBR-R809, Houston, TX 77030, USA. Tel: +713 798 5370; Fax: +713 798 7418; E-mail: pawels@bcm.edu

6 These authors contributed equally to this work.
}

Received 19 June 2014; revised 21 August 2014; accepted 5 September 2014; published online 15 October 2014 


\section{MATERIALS AND METHODS}

\section{Patients}

Patient 1 is a 13 -year-old boy, former 28 -week preemie, with autistic behavior, developmental delay, attention deficit hyperactivity disorder (ADHD), obsessive-compulsive disorder (OCD), anxiety, verbal language delay, dyslexia, and a history of behavioral problems. He is short statured (takes growth hormone) and his head circumference is $55 \mathrm{~cm}$ ( $50 \%$ percentile). He has hypertension treated with lisinopril. The CDKL5 duplication was inherited from the mother, who reported to have problems with reading and mathematics while at school. Her head circumference is $57 \mathrm{~cm}$.

Patient 2 is a 6-year-old girl with developmental delay, obesity, and sleeping problems (jerks/twitches, snoring/breathing problems). She had language delay and had done poorly in learning colors, numbers, and words. She has numerous behavioral problems including sadness, irritability, inability to get along with others, and taking things that do not belong to her. At 4.5 years of age, her height and weight were above 95th percentile. She is also macrocephalic. Her father was unavailable for clinical evaluation.

Patient 3 was evaluated between 6-9 years of age for mild intellectual disability, developmental regression, speech impairment, ADHD, and selfinjurious and stereotyped behaviors. He was born at 36 weeks. The umbilical cord was wrapped around his neck at birth; he experienced difficulty in breathing and had low Apgar scores. He had developmental regression around 1 year when he had a few words and stopped speaking. He has significant echolalia and speech impairment as well as trouble with fine motor skills, specifically with handwriting and using utensils to eat. He also presents with obsessive-compulsive as well as autistic behaviors and often flaps his hands. He favors routines, patterns, lining things up, and does not like transitions. He is very argumentative, manifests oppositional behaviors, often gets aggressive with hitting other children and banging his own head. He licks things, is sensitive to certain textures of foods and is highly resistant to pain. He is normocephalic with mildly deeply placed eyes, mild frontal prominence and thin upper lips. His head MRI and EEG were normal. Based on DSM IV criteria, he was diagnosed with Asperger syndrome, ADHD, and sensory integration disorder. Family history is consistent for bipolar disorder in two maternal uncles, ADHD and learning disabilities in his brother and sister, depression in the maternal grandfather, and learning disabilities in his mother and maternal grandmother. He has gross motor delay consistent with sitting at 8 months and walking at 15 months. The Xp22 duplication was inherited from the mother.

Patient 4 is a 14-year-old boy referred for evaluation because of slightly delayed development. He walked at 18 months and had his first words at 2 years of age. He received speech and physical therapy starting at 18 months until 3 years of age. He seems to be always fatigued and is sometimes easily distracted; however, no diagnosis of ADHD has been made. He has been homeschooled, and is completing the 7th grade. His mother reports that he has consistently performed at or above grade level; however, he has had issues with becoming confused while performing multiple step tasks. He gets easily distracted. He has problems with gross motor activity and has difficulty writing for school work activities. He was also found to have enlarged aorta, mixed pectus deformity that does not require surgery, tall stature, macrocephaly, hyperextensibility, pes planus, and facial asymmetry. He also has a history of neuroblastoma of right adrenal gland. His height was greater than the 97th percentile, his weight was between the 90th and 97th percentile, and his head circumference was greater than two SD (FOC $56 \mathrm{~cm}$ ). At 13 years of age, he had tall stature, malar hypoplasia, pectus carinatum and excavatum, slight scoliosis, arachnodactyly, bilateral thumb and wrist signs, hind foot deformities, and dilated aorta, sufficient to meet clinical diagnosis for Marfan syndrome. Parents have refused further genetic testing to confirm the Marfan syndrome diagnosis.

Patient 5 was referred at age 6 years for evaluation of speech impairment, fine motor delay, hyperactivity, and stereotypical behaviors. He was adopted at age 2 weeks; his biological mother was suspected to be on cocaine, marijuana, and antidepressants. He had normal gross motor milestones, but exhibits fine motor delay consistent with poor pencil grasp and difficulty with handwriting. He had mild speech delay and communication impairment but has caught up and now has a huge vocabulary with echolalia. He is hyperactive, has problems with social skills, pushes other children and bites his mother and father. He has hand flapping and stereotypic movements, as well as rocking body movements.
$\mathrm{He}$ is sensitive to smells and textures of clothes. He likes routines and is obsessed with doors. He likes deep pressure and has sensory integration problems. Based on DSM IV criteria, he was diagnosed with Asperger syndrome and sensory integration disorder. He has macrocephaly $(54.5 \mathrm{~cm}$, $>95$ th percentile for age). G-banded chromosomal analysis, fragile $\mathrm{X}$ and PTEN testing, and brain MRI imaging were all normal. He is on ADHD medications (Intuniv).

Patient 6 was assessed between $6-8$ years of age for intellectual disability and speech impairment. She was born via Cesarean section secondary to breech presentation. Her mother had contractions at 6 months that resolved on their own. She had delayed head control and started to walk around 16 months. She is unable to speak in full sentences, her vocabulary is about 30-40 words and she has a lot of nonsensical words. She has fine motor delay consistent with handwriting difficulties. She had a surgery for exotropia and has poor eye contact which might also be attributed to her eye problems. She had poor social skills but has improved now. She does not like to share. She also has memory problems and some cognitive deficits. She flaps her hands often and laughs inappropriately. She is hyperactive, has autistic traits and likes to line up things, and spin in circles. She has a texture preference to foods consistent with sensory integration problems. She likes routines and has repetitive behaviors. She was normocephalic on exam. Her brain MRI is significant for right tonsillar ectopia. She was diagnosed with autism, intellectual disability, and speech impairment based on DSM IV criteria. She had a normal G-banded chromosome analysis, and MECP2 sequencing. Her mother, who has the CDKL5 duplication, is reportedly healthy. Family history was significant for a maternal cousin with probable intellectual disability.

Patient 7 is an 8-year-old Caucasian female, a product of a 36-week uneventful pregnancy and labor, which was induced because of high blood pressure. Birth measurements included a weight of $6 \mathrm{lbs} 11 \mathrm{oz}$. She did not latch on and had a poor suck, but did gain weight with bottle feedings. Feedings improved at 6 months. She had poor muscle tone and was sitting at 1 year, walking at 2.5 years, and talking at 2.5 years. Speech and occupational therapy began at 3 years of age. She has occasional defiance and aggression, which tends to occur when there are sudden changes in her routine. She has a strong need for maintaining her order and routine, and if changes occur she becomes very frustrated and acts out when her routine is changed. She has very rapid mood swings going from calm to angry with screaming and ranting, but she does not hit, bite or throw things. She loves to stack cups and then take them down and will repeat this routine for 30-60 min. She will flip through the pages of a book over and over for an hour at a time. She has less sucking-of-fingers behavior, but is now picking at her ears (sometimes till they bleed). There has been no regression, learning plateau, or episodic encephalopathy. She has glasses for astigmatism. She may have had a seizure with fever at age 3.5 years (EEG was normal). She has an increased pain tolerance. Her weight is $33.5 \mathrm{~kg}(90-95 \%)$, height $119.8 \mathrm{~cm}(10 \%)$, BMI 23.34 (>97\%), and FOC $52.4 \mathrm{~cm}$ (normal). Index and fifth fingers had short tapering distal phalanges with mildly hypoplastic creases. She has brachycephaly, a wide, flattened face, mild frontal bossing and deeply set eyes, prominent ears with mild cupping, a thin upper and outpouched lower lip with inverted $\mathrm{V}$ shape to lips, an accessory nipple bilaterally, calluses on her fingers from sucking and chewing, café-au-lait spots (one on left thigh $>1.5 \mathrm{~cm}$ and on left buttock) and hypopigmented spots near each scapula (each $>1 \mathrm{~cm}$ ). The neck was supple with full range of motion and with no goiter. She has a low-pitched hoarse voice. Her urine has an abnormal odor. Her diagnosis includes intellectual disability, seemingly functioning at about a 5.5 year level though there are performance gaps, and autistic spectrum disorder. She has a brother and a maternal first cousin with schizophrenia and bipolar disease. Her carrier mother is healthy. A 4-generation pedigree is otherwise unremarkable.

Patients' clinical features are summarized in Table 1.

\section{Array CGH analyses}

Genomic DNA was extracted from peripheral blood via the Puregene DNA isolation kit (Gentra System, Minneapolis, MN, USA). Array CGH (aCGH) was performed using custom-designed exon targeted oligonucleotide microarrays (V8.0 OLIGO, V8.1.1 OLIGO, V8.2 OLIGO HR+SNP, V8.3 OLIGO HR+SNP, $180 \mathrm{~K}$, and V9.1.1 OLIGO HR+SNP, $400 \mathrm{~K}$ ) that were designed by the Medical 
Table 1 Clinical characteristics of individuals with CDKL5 duplication and deficiency

\begin{tabular}{|c|c|c|c|c|c|c|c|c|c|c|c|c|}
\hline & Pt 1 & Pt 1's mother & Pt 2 & Pt 3 & $\begin{array}{l}\text { Pt 3's } \\
\text { mother }\end{array}$ & Pt 4 & Pt 5 & Pt 6 & $\begin{array}{l}\text { Pt 6's } \\
\text { mother }\end{array}$ & Pt 7 & $\begin{array}{l}\text { Pt 7's } \\
\text { mother }\end{array}$ & CDKL5 deficiency \\
\hline Sex & M & $\mathrm{F}$ & $\mathrm{F}$ & M & $\mathrm{F}$ & M & M & $\mathrm{F}$ & $\mathrm{F}$ & $\mathrm{F}$ & $\mathrm{F}$ & \\
\hline Age (years) & 13 & $\mathrm{~N} / \mathrm{A}$ & 6 & 9 & 36 & 14 & 6 & 8 & 31 & 8 & 36 & \\
\hline Minimal duplication size (kb) & 935 & 935 & 935 & 789 & 789 & 540 & 642 & 642 & 643 & 635 & 635 & \\
\hline Duplication inheritance & Mat & Unk & Pat & Mat & Unk & Unk & Unk & Mat & Unk & Mat & Unk & \\
\hline $\begin{array}{l}\text { Developmental delay/ } \\
\text { Intellectual disability }\end{array}$ & + & $\begin{array}{l}\text { Reading \& math } \\
\text { problems }\end{array}$ & + & + & $\begin{array}{l}\text { Learning } \\
\text { problems }\end{array}$ & + & + & + & - & + & - & + \\
\hline Developmental regression & - & $\mathrm{N} / \mathrm{A}$ & - & + & $N / A$ & - & - & - & - & - & - & + \\
\hline Hypotonia & - & $\mathrm{N} / \mathrm{A}$ & - & - & $N / A$ & - & - & - & - & + & - & + \\
\hline Seizures & - & $\mathrm{N} / \mathrm{A}$ & - & - & $N / A$ & - & - & - & - & - & $-(?)$ & Early onset, intractable \\
\hline Autism spectrum disorder & + & $\mathrm{N} / \mathrm{A}$ & - & + & $\mathrm{N} / \mathrm{A}$ & - & + & + & - & + & - & - \\
\hline Sensory integration disorder & - & $\mathrm{N} / \mathrm{A}$ & - & + & $\mathrm{N} / \mathrm{A}$ & - & + & + & - & + & - & - \\
\hline $\begin{array}{l}\text { Attention deficit hyperactivity } \\
\text { disorder }\end{array}$ & + & $\mathrm{N} / \mathrm{A}$ & - & + & $\mathrm{N} / \mathrm{A}$ & - & + & + & - & - & - & - \\
\hline $\begin{array}{l}\text { Obsessive-compulsive } \\
\text { disorder }\end{array}$ & + & $\mathrm{N} / \mathrm{A}$ & - & + & $N / A$ & - & + & - & - & - & - & - \\
\hline $\begin{array}{l}\text { Speech impairment/language } \\
\text { delay }\end{array}$ & + & $\mathrm{N} / \mathrm{A}$ & + & + & $N / A$ & + & + & + & - & + & - & + \\
\hline Behavioral abnormalities & + & $\mathrm{N} / \mathrm{A}$ & + & + , stereotypic & $N / A$ & - & + & + & - & + , stereotypic & - & Hand stereotypies \\
\hline Sleep difficulties & - & $\mathrm{N} / \mathrm{A}$ & + & - & $N / A$ & - & - & - & - & - & - & - \\
\hline Resistance to pain & - & $\mathrm{N} / \mathrm{A}$ & - & + & $N / A$ & - & - & - & - & + & - & - \\
\hline Macrocephaly & - & - & + & - & $N / A$ & + & + & - & - & - & - & Microcephaly \\
\hline Dysmorphic features & - & N/A & - & + & $N / A$ & + & - & - & - & + & - & - \\
\hline Obesity & - & $\mathrm{N} / \mathrm{A}$ & + & - & $N / A$ & - & - & - & - & + & - & Dysphagia \\
\hline
\end{tabular}

Abbreviations: Mat, maternal; N/A, not available; Pat, paternal; Unk, unknown.

Genetics Laboratories at Baylor College of Medicine (http://www.bcm.edu/ geneticlabs/) and manufactured by Agilent Technologies (Santa Clara, CA, USA) as described. ${ }^{35,36}$ The genomic coordinates of Xp22 duplication breakpoints have been submitted to ClinVar database (http://ncbi.nlm.nih.gov/ clinvar/) where they can be accessed with numbers SCV000120161-6.

\section{FISH analyses}

Confirmatory fluorescence in situ hybridization (FISH) analyses were performed using BAC clones RP11-106N3 (pts 1 and 4), and RP11-558P14 (pt 2) on chromosome X and RP11-194J3 (pt 2) on chromosome 17q25.1 using the standard procedures.

\section{Long-range PCR and DNA sequence analyses}

The junction fragments of the duplications were amplified using long-range PCR with Takara LA Taq Polymerase (TaKaRa Bio USA, Madison, WI, USA), according to the manufacturer's instructions. Primers were designed using Primer3 software (http://frodo.wi.mit.edu/primer3/) at the apparent head-totail duplication boundaries inferred from the aCGH analysis. PCR products were visualized on a $1 \%$ agarose gel, purified with ExoSAP-IT (USB, Cleveland, $\mathrm{OH}, \mathrm{USA}$ ) to remove unconsumed dNTPs and primers, and directly sequenced by the Sanger method (Lone Star Labs, Houston, TX, USA).

\section{$\mathrm{X}$-Inactivation studies}

X-Inactivation (XCI) studies were performed as described ${ }^{37}$ with slight modification. Genomic DNA was extracted from the blood specimens, and PCR was used to amplify a region of the androgen receptor gene containing the CAG polymorphism. To determine the extent of inactivation of each allele, one sample was digested with HpaII. Fluorescent-labeled primers were used, and the amplification products were separated by capillary gel electrophoresis. The products were then analyzed using the Genescan software. Calculation of the ratio of the two alleles after digestion was performed using a correction for the preferential amplification of one of the two alleles in the undigested sample.

\section{In silico DNA sequence analyses}

Genomic sequences determined on the basis of oligonucleotide coordinates from aCGH experiments were downloaded from the UCSC genome browser (NCBI build 37/hg19, http://genome.ucsc.edu/) and assembled using Sequencher 4.8 software (Gene Codes Corporation, Ann Arbor, MI, USA). Interspersed repeat sequences were identified using RepeatMasker (http://www. repeatmasker.org/).

\section{RESULTS}

\section{Array CGH analyses}

By using chromosomal microarray analysis (CMA; versions 8 and 9) in 27000 patients, we identified seven unrelated families with submicroscopic duplications at Xp22, involving CDKL5 (Table 2; Figure 1). The minimal duplication size ranged from $\sim 540 \mathrm{~kb}$ to $\sim 935$ $\mathrm{kb}$, and the genomic position of the breakpoints in each individual was determined as follows: Patient 1: chrX.hg19:g.18,221,537_19,156, 151dup; Patient 2: chrX.hg19:g.18,221,537_19,156,151dup; Patient 3: chrX.hg19:g.18,367,552_19,156,151dup; Patient 4: chrX.hg19:g.18,336, 700_18,876,286dup; Patient 5: chrX.hg19:g.18,460,072_19,101,792dup; Patient 6: chrX.hg19:g.18,460,072_19,101,792dup; and Patient 7: chrX. hg19:g.18,460,071_19,094,977dup.

In addition, CMA revealed an $\sim 453 \mathrm{~kb}$ duplication on chromosome $17 \mathrm{q} 25.1$ in patient 2 , an $\sim 402 \mathrm{~kb}$ duplication on 9 p24.1 in patient 3 (inherited from the healthy father), and an $\sim 565 \mathrm{~kb}$ deletion on chromosome $16 \mathrm{q} 21$ in patient 4 . Given that these CNVs overlap with multiple deletions and duplications deposited in the Database of Genomic Variants, they were interpreted as likely benign. Xp22 duplications in patients 1, 2 and 4, and 17q25.1 duplication in patient 2 were verified by FISH.

Parental testing showed that the Xp22 duplications were inherited from the mother in four patients $(1,3,6$, and 7$)$ and from the father in one patient (2). Samples of biological parents for patients 4 and 5 were unavailable. 
Long-range PCR and DNA sequence analyses

In patient 5, DNA sequencing of the junction fragment amplified using LR-PCR with primers, F: 5'-ATAACAAGCCATAAGAAGTG GCTCGCTTTACT-3' and R: 5'-GGATATGATACCTCAACTTCC TGTAGGGTCTT-3' revealed a complex nature of the $\sim 667 \mathrm{~kb}$ duplication in Xp22.13 (Figure 2). The distal duplication breakpoint maps within the AluSx3 element at 18,392,778(786) and the proximal breakpoint within the AluSx1 element at chrX:19,124,907(915) with 7 bp GGCACCT microhomology. In addition, $\sim 1.4 \mathrm{~kb}$ proximal from the distal breakpoint, we identified a second junction fragment, resulting from a loss of $\sim 64 \mathrm{~kb}$ genomic interval 18,394,206$18,459,045$, mapping $1.348 \mathrm{bp}$ upstream to the noncoding exon $1 \mathrm{a}$, between two nonhomologous fragments of the ERV1 elements, and including a $13 \mathrm{bp}$ insertion of $(\mathrm{AC})_{6} \mathrm{~A}$ (Figure 2).

The identical-sized double junction fragments were also identified in patients 6 and 7 of different ethnicities (Supplementary Figure 1). DNA sequencing of these three fragments showed no sequence differences, indicating that this ancestral complex duplication is identical by descent and likely arose several generations ago as a result of DNA template switching replication error. ${ }^{38}$ Alternatively, the $64 \mathrm{~kb}$ deletion between the HERV elements might have arisen owing to polymerase slippage error during or following the formation of CDKL5 duplication.

\section{Public CNV databases}

Decipher and ISCA CNV database query revealed only one smallersized CDKL5 duplication in a Decipher XX female patient 4160. This duplication harbors only the $3^{\prime}$ portion of CDKL5, excluding the translation initiation codon present in exon 2 , and thus is rather unlikely to result in an increased dosage of CDKL5.

\section{XCI studies}

XCI pattern studied in patient 1's mother, patient 2, and patients 6 and 7 and their mothers was found as random in all of them (Supplementary Table 1).

\section{DISCUSSION}

In contrast to $M E C P 2$ deficiency, the severity of clinical manifestations due to CDKL5 deficiency does not differ significantly between males and females. ${ }^{18,39-41}$ The vast majority of genetic alterations in CDKL5

Table 2 Results of CMA and FISH analyses in patients with Xp22 duplications

\begin{tabular}{|c|c|c|c|c|}
\hline Pt & t Array & CMA-determined minimal duplication region on Xp22.13 & $\begin{array}{l}\text { CMA-determined maximal duplication } \\
\text { region on Xp22.13 }\end{array}$ & Additional reported $\mathrm{CNV}$ \\
\hline 1 & $\begin{array}{l}\text { V8.1.1 OLIGO, } \\
180 \mathrm{~K}\end{array}$ & $\begin{array}{l}\text { arr Xp22.13(chrX:18,221,537-19,156,151)x2. nuc ish } \\
\text { Xp22.13(RP11-106N3×2)mat }\end{array}$ & chrX:18,177,897-19,208,040 & N/A \\
\hline 2 & $\begin{array}{l}\text { V8.0 OLIGO, } \\
180 \mathrm{~K}\end{array}$ & $\begin{array}{l}\text { arr Xp22.13(chrX:18,221,537-19,156,151)x3. nuc ish } \\
\text { Xp22.13(RP11-558P14x3)pat }\end{array}$ & 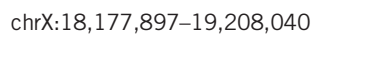 & $\begin{array}{l}\text { arr 17q25.1(72,205,982-72,659,034)x3. nuc ish } \\
17 q 25.1(R P 11-194 J 3 \times 3) \text { pat }\end{array}$ \\
\hline 3 & $\begin{array}{l}\text { V8.3 OLIGO } \\
\text { HR+SNP, } 180 \mathrm{~K}\end{array}$ & arr Xp22.13(18,367,552-19,156,151)x2mat & 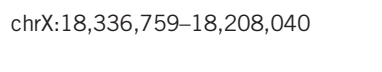 & arr 9p24.1(6,575,731-6,977,999)x3pat \\
\hline 4 & $\begin{array}{l}\text { V8.1.1 OLIGO, } \\
180 \mathrm{~K}\end{array}$ & arr Xp22.13(chrX:18,336,700-18,876,286)x2 & 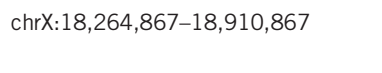 & arr $16 q 21(63,171,157-63,736,645) \times 1$ \\
\hline 5 & $\begin{array}{l}\text { V9.1.1 OLIGO } \\
\text { HR+SNP, } 400 \mathrm{~K}\end{array}$ & arr Xp22.13(18,460,072-19,101,792)x2 & 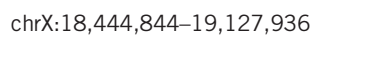 & $\mathrm{N} / \mathrm{A}$ \\
\hline 6 & $\begin{array}{l}\text { V9.1.1 OLIGO } \\
\text { HR+SNP, } 400 \mathrm{~K}\end{array}$ & arr Xp22.13(18,460,072-19,101,792)x2mat & 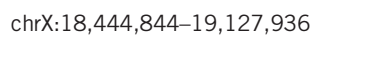 & $\mathrm{N} / \mathrm{A}$ \\
\hline 7 & $\begin{array}{l}\text { V8.2 OLIGO } \\
\text { HR+SNP, } 180 \mathrm{~K}\end{array}$ & arr Xp22.13(18,460,071-19,094,977)x3mat & chrX:18,444,844-19,127,936 & $\mathrm{N} / \mathrm{A}$ \\
\hline
\end{tabular}

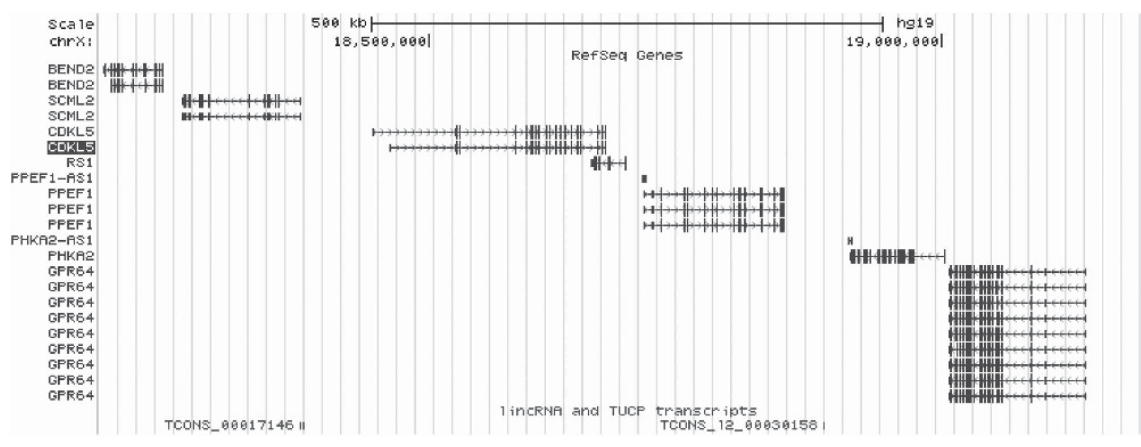

pt 1, pt 2

pt 3

pt 4

pt 5, pt 6, pt 7

Figure 1 Schematic representation of Xp22 duplications encompassing CDKL5 in patients 1-7. 
a
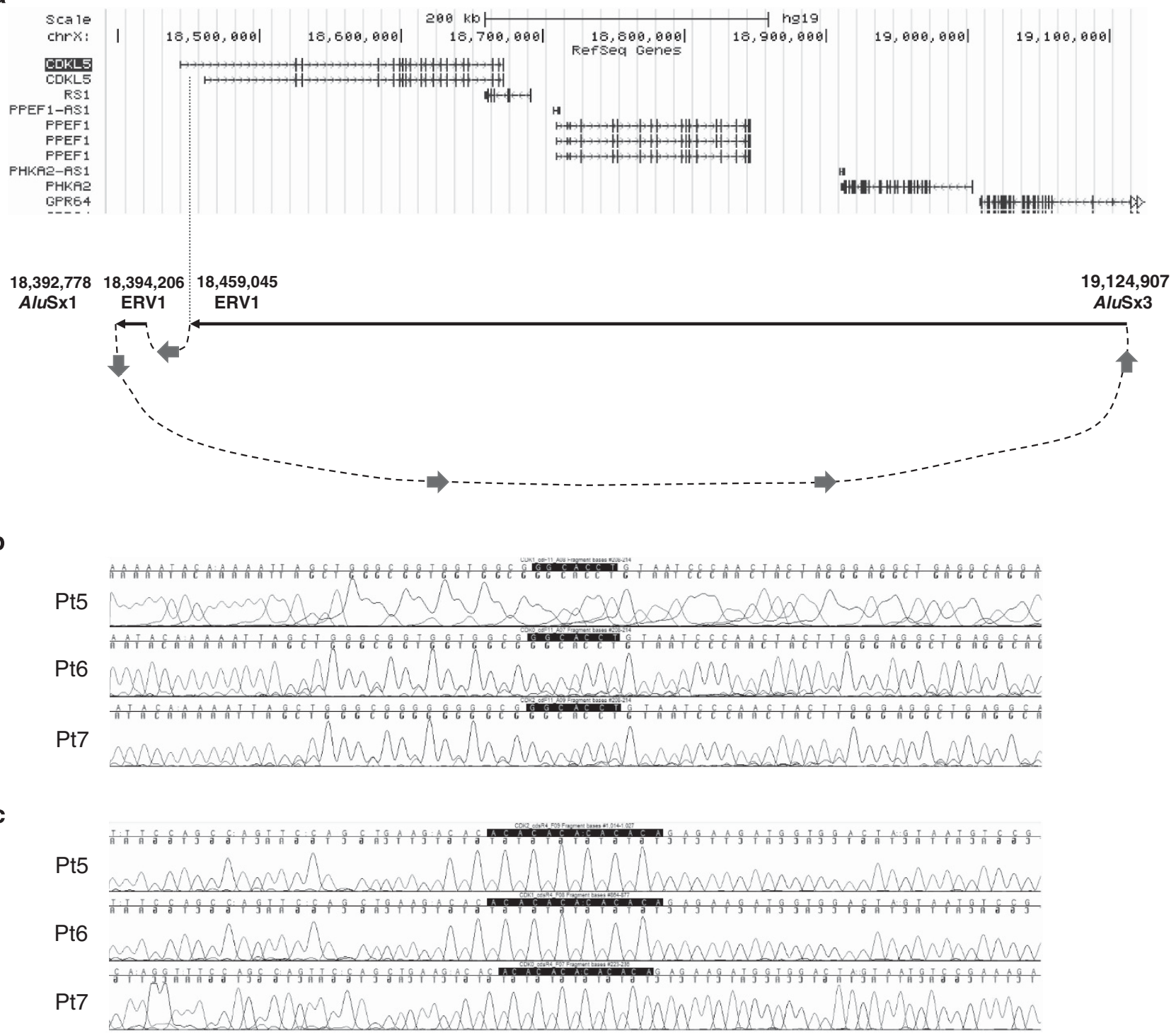

Figure 2 Identical duplications in patients 5-7 harbor only the short CDKL5 isoform expressed in the fetal brain. (a) A complex structure of the duplications is shown. Duplication breakpoints map within AluSx elements (b) and the associated deletion breakpoints map within ERV1 elements (c).

have been reported in female patients with a random XCI pattern in the analyzed blood DNA. ${ }^{42-44}$ Sartori et a ${ }^{45}$ reported a CDKL5 alteration in a 47,XXY boy with the early-onset seizure variant of Rett syndrome and concluded that a random pattern of XCI did not influence the severity of the disease. Skewed XCI has been proposed to explain the observed phenotypic variability in some females heterozygous for a mutated or deleted CDKL5 allele. The most severe phenotype with early-onset encephalopathy was described in the females with the balanced X;autosome translocations, wherein the normal X chromosome was completely inactivated. ${ }^{46}$ In our cohort, all females with CDKL5 duplications had random XCI pattern; however, the mothers of male patients 1 and 3 were mildly affected when compared with their sons, and mothers of patients 6 and 7 were healthy, suggesting that their XCI pattern may be different in blood and brain.

CDKL5 isoform I, transcribed from exon 1, is expressed in a wide range of brain compartments, including cerebral cortex, hippocampus, cerebellum, striatum, and brain stem, as well as in testes and thymus. ${ }^{20}$
CDKL5 levels were found to be low in the embryonic cortex and strongly induced at perinatal and postnatal stages in maturing neurons residing in both cerebral cortex and hippocampus until day P14, where after it declines. ${ }^{12}$ A detailed analysis of Cdkl5 expression in adult mouse brain (Allen Brain Atlas) showed that its mRNA levels are particularly high in the adult forebrain, ${ }^{13}$ consistent with its role in neuronal maturation. ${ }^{12}$

Conversely, isoform II, transcribed from exons 1a and $1 \mathrm{~b}$ (but not exon 1), is only expressed at very low levels in human fetal brain and testes. ${ }^{46,47}$ It remains to be determined in what brain compartment(s) and in which cells this isoform is predominantly expressed. The phenotypic similarities observed in male patient 5 and female patients 6 and 7 with the duplication of CDKL5 isoform II may result from abnormalities in spatiotemporal functions or interactions of CDKL5 during fetal brain development. In support of this notion, expression of $C d k l 5$ in mouse brain overlaps with that of Mecp2 during neural maturation and synaptogenesis. ${ }^{4,19}$ 
Mari et al $^{19}$ found that CDKL5 and MECP2 interact both in vitro and in vivo and that CDKL5 mediates MECP2 phosphorylation in vitro. However, Lin et al ${ }^{20}$ demonstrated that this phosphorylation might have resulted from trace amounts of nonspecific kinases in the co-immunoprecipitate. Nonetheless, using an in vitro kinase assay with epitope-tagged proteins expressed in HEK293 cells, Williamson et al ${ }^{23}$ found that $107 \mathrm{kDa}$ and $115 \mathrm{kDa}$ isoforms of CDKL5 phosphorylated MECP2 and underwent autophosphorylation. Carouge et a ${ }^{22}$ proposed that MECP2 represses transcription of CDKL5, likely through methylation of the CpG island overlapping CDKL5 exon 1. Further studies are necessary to elucidate these putative interactions.

Interestingly, in contrast to $55 \%$ of patients with CDKL5 genomic deletions or point mutations who have acquired progressing microcephaly, ${ }^{7,38,48,49}$ our patients 2,4 , and 5 had macrocephaly. Similar micro- $v s$ macrocephaly phenomenon has been reported in patients with recurrent deletions and reciprocal duplications in genomic regions $1 \mathrm{q} 21.1$ and $16 \mathrm{p} 11.2$. Brunetti-Pierri et a ${ }^{50}$ reported 21 patients with a recurrent $1.35 \mathrm{Mb}$ deletion and 15 patients with reciprocal duplication in 1q21.1. The deletion and duplication were associated with microcephaly and macrocephaly, respectively. A reverse correlation was described in patients with recurrent $\mathrm{CNVs}$ in 16p11.2. ${ }^{51}$ The macro- and microcephaly observed in patients with CDKL5 duplications and deficiency, respectively, corroborate a diametric model of autism spectrum and psychotic spectrum behavioral phenotypes in genomic sister disorders. ${ }^{52}$

Unlike CDKL5 deficiency, none of the presented patients with duplication had epilepsy and patients 3 and 7 had normal EEGs. Brain imaging in patients with CDKL5 mutations demonstrated cortical atrophy and white-matter hyperintensities. ${ }^{53}$ In patients 3 and 5, MRI studies were normal and patient 6 had right tonsillar ectopia. Interestingly, patient 6 has also exotropia, which is frequently seen in patients with CDKL5 deficiency.

The contribution of other genes in the duplicated fragments to the described patient phenotypes is less likely. None of the protein-coding and putative long non-coding RNA genes from the minimum duplication region (Figure 1) has increased expression in the brain or has been linked to the brain development or neurobehavioral phenotype. Alterations in the Retinoschisin gene (RS1) have been described in patients with X-linked retinoschisis (MIM 312700), leading to reduced visual acuity in affected subjects. Mutations in PHKA2 have been reported in patients with X-linked hepatic glycogen storage disease (GSD9A; MIM 306000). Thus far, no disease-related duplications of PHKA2 or RS1 have been described.

In aggregate, we propose that the increased dosage of CDKL5 might have affected global output of the interactions of the CDKL5 kinase with its substrates, leading to perturbation of synaptic plasticity and development; manifestations of this perturbation include difficulties in learning, autistic and hyperactive behavior, developmental and speech delay, and macrocephaly.

\section{CONFLICT OF INTEREST}

The Department of Molecular and Human Genetics at Baylor College of Medicine offers extensive genetic laboratory testing, including chromosomal microarray analysis, and derives revenue from this activity. The remaining authors declare no conflict of interest.

\section{ACKNOWLEDGEMENTS}

We thank Huda Zoghbi and Jozef Gecz for helpful discussion, and the families for participating in the study.

\section{DISCLAIMER}

We confirm that we have read the Journal's position on issues involved in ethical publication and affirm that this report is consistent with those guidelines.

1 Amir RE, Van den Veyver IB, Wan M et al: Rett syndrome is caused by mutations in X-linked MECP2, encoding methyl-CpG-binding protein 2. Nat Genet 1999; 23: $185-188$.

2 Miltenberger-Miltenyi G, Laccone F: Mutations and polymorphisms in the human methyl CpG-binding protein MECP2. Hum Mutat 2003; 22: 107-115.

3 Neul JL, Kaufmann WE, Glaze DG et al: Rett syndrome: revised diagnostic criteria and nomenclature. Ann Neurol 2010; 68: 944-950.

4 Weaving LS, Christodoulou J, Williamson SL et al: Mutations of CDKL5 cause a severe neurodevelopmental disorder with infantile spasms and mental retardation. Am J Hum Genet 2004; 75: 1079-10935.

5 Tao J, Van Esch H, Hagedorn-Greiwe $M$ et al: Mutations in the X-linked cyclindependent kinase-like 5 (CDKL5/STK9) gene are associated with severe neurodevelopmental retardation. Am J Hum Genet 2004; 75: 1149-1154.

6 Bienvenu T, Chelly J: Molecular genetics of Rett syndrome: when DNA methylation goes unrecognized. Nature Rev Genet 2006; 7: 415-426, Note: Erratum: Nat Rev Genet 2006; 7: 583

7 Russo S, Marchi M, Cogliati F et al: Novel mutations in the CDKL5 gene, predicted effects and associatedphenotypes. Neurogenetics 2009; 10: 241-250.

8 Rademacher N, Hambrock M, Fischer U et al: Identification of a novel CDKL5 exon and pathogenic mutations in patients with severe mental retardation, early-onset seizures and Rett-like features. Neurogenetics 2011; 12: 165-167.

9 Ariani F, Hayek G, Rondinella D et al: FOXG1 is responsible for the congenital variant of Rett syndrome. Am J Hum Genet 2008; 83: 89-93.

10 Mencarelli MA, Spanhol-Rosseto A, Artuso $\mathrm{R}$ et al: Novel FOXG1 mutations associated with the congenital variant of Rett syndrome. J Med Genet 2010; 47 49-53.

11 Philippe C, Amsallem D, Francannet C et al: Phenotypic variability in Rett syndrome associated with FOXG1 mutations in females. J Med Genet 2010; 47: 59-65.

12 Rusconi L, Salvatoni L, Giudici L et al: CDKL5 expression is modulated during neuronal development and its subcellular distribution is tightly regulated by the $\mathrm{C}$-terminal tail. J Biol Chem 2008; 283: 30101-30111.

13 Kilstrup-Nielsen C, Rusconi L, La Montanara P et al: What we know and would like to know about CDKL5 and its involvement in epileptic encephalopathy. Neural Plast 2012; 2012: 1-12.

14 Van Esch H, Jansen A, Bauters $M$ et al: Encephalopathy and bilateral cataract in a boy with an interstitial deletion of Xp22 comprising the CDKL5 and NHS genes. Am J Med Genet A 2007; 143: 364-369.

15 Erez A, Patel AJ, Wang X et al: Alu-specific microhomology-mediated deletions in CDKL5 in females with early-onset seizure disorder. Neurogenetics 2009; 10: 363-369.

16 Bartnik M, Derwinska K, Gos M et al: Early-onset seizures due to mosaic exonic deletions of CDKL5 in a male and two females. Genet Med 2011; 13: 447-452.

17 Bahi-Buisson N, Girard B, Gautier A et al: Epileptic encephalopathy in a girl with an interstitial deletion of Xp22 comprising promoter and exon 1 of the CDKL5 gene. Am J Med Genet B Neuropsychiatr Genet 2009; 153B: 202-207.

18 Mei D, Marini C, Novara F et al: Xp22.3 genomic deletions involving the CDKL5 gene in girls with early onset epileptic encephalopathy. Epilepsia 2010; 51 647-654.

19 Mari F, Azimonti S, Bertani I et al: CDKL5 belongs to the same molecular pathway of $\mathrm{MeCP} 2$ and it is responsible for the early-onset seizure variant of Rett syndrome. Hum Mol Genet 2005; 14: 1935-1946.

20 Lin C, Franco B, Rosner MR: CDKL5/Stk9 kinase inactivation is associated with neuronal developmental disorders. Hum Mol Genet 2005; 14: 3775-3786.

21 Bertani I, Rusconi L, Bolognese $\mathrm{F}$ et al: Functional consequences of mutations in CDKL5, an X-linked gene involved in infantile spasms and mental retardation. J Biol Chem 2006; 281: 32048-32056.

22 Carouge D, Host L, Aunis D et al: CDKL5 is a brain MeCP2 target gene regulated by DNA methylation. Neurobiol Dis 2010; 38: 414-424.

23 Williamson SL, Giudici L, Kilstrup-Nielsen C et al: A novel transcript of cyclindependent kinase-like 5 (CDKL5) has an alternative $\mathrm{C}$-terminus and is the predominant transcript in brain. Hum Genet 2012; 131: 187-200.

24 Livide G, Patriarchi T, Amenduni M et al: GluD1 is a common altered player in neuronal differentiation from both MECP2-mutated and CDKL5-mutated iPS cells. Eur J Hum Genet 2014; doi: 10.1038/ejhg.2014.81. (epub ahead of print).

25 Van Esch H, Bauters M, Ignatius J et al: Duplication of the MECP2 region is a frequent cause of severe mental retardation and progressive neurological symptoms in males. Am J Hum Genet 2005; 77: 442-453.

26 Ramocki MB, Peters SU, Tavyev YJ et al: Autism and other neuropsychiatric symptoms are prevalent in individuals with MECP2 duplication syndrome. Ann Neurol 2009; 66 : 771-782

27 Yeung A, Bruno D, Scheffer IE et al: $4.45 \mathrm{Mb}$ microduplication in chromosome band $14 q 12$ including FOXG1 in a girl with refractory epilepsy and intellectual impairment. Eur J Med Genet 2009; 52: 440-442. 
28 Brunetti-Pierri N, Paciorkowski AR, Ciccone R et al: Duplications of FOXG1 in 14q12 are associated with developmental epilepsy, mental retardation, and severe speech impairment. Eur J Hum Genet 2011; 19: 102-107.

29 Striano P, Paravidino R, Sicca F et al: West syndrome associated with $14 q 12$ duplications harboring FOXG1. Neurology 2011; 76: 1600-1602.

30 Tohyama J, Yamamoto T, Hosoki $\mathrm{K}$ et al: West syndrome associated with mosaic duplication of FOXG1 in a patient with maternal uniparental disomy of chromosome 14. Am J Med Genet 2011; 155: 2584-2588.

31 Froyen G, Van Esch $\mathrm{H}$, Bauters $\mathrm{M}$ et al. Detection of genomic copy number changes in patients with idiopathic mental retardation by high-resolution $\mathrm{X}$-arrayCGH: important role for increased gene dosage of XLMR genes. Hum Mutat 2007; 28: 1034-1042.

32 Tzschach A, Chen W, Erdogan $F$ et al. Characterization of interstitial Xp duplications in two families by tiling path array CGH. Am J Med Genet A 2008; 146A: 197-203.

33 Thorson L, Bryke C, Rice G et al. Clinical and molecular characterization of overlapping interstitial Xp21-p22 duplications in two unrelated individuals. Am J Med Genet $A$ 2010; 152A: 904-915.

34 Sismani C, Anastasiadou V, Kousoulidou L et al. $9 \mathrm{Mb}$ familial duplication in chromosome band Xp22.2-22.13 associated with mental retardation, hypotonia and developmental delay, scoliosis, cardiovascular problems and mild dysmorphic facial features. Eur J Med Genet 2011; 54: e510-e515.

35 Boone PM, Bacino CA, Shaw CA et al: Detection of clinically relevant exonic copy-number changes by array CGH. Hum Mutat 2010; 31: 1326-1342.

36 Wiszniewska J, Bi W, Shaw C et al: Combined array CGH plus SNP genome analyses in a single assay for optimized clinical testing. Eur J Hum Genet 2014; 22: 79-87.

37 Allen RC, Zoghbi HY, Moseley AB et al: Methylation of Hpall and Hhal sites near the polymorphic CAG repeat in the human androgen-receptor gene correlates with $\mathrm{X}$ chromosome inactivation. Am J Hum Genet 1992; 51: 1229-1239.

38 Hastings PJ, Ira G, Lupski JR: A microhomology-mediated break-induced replication model for the origin of human copy number variation. PLoS Genet 2009; 5: e1000327.

39 Bahi-Buisson N, Nectoux J, Rosas-Vargas $\mathrm{H}$ et al: Key clinical features to identify girls with CDKL5 mutations. Brain 2008; 131: 2647-2661.
40 Nemos C, Lambert L, Giuliano F et al: Mutational spectrum of CDKL5 in early-onset encephalopathies: a study of a large collection of French patients and review of the literature. Clin Genet 2009; 76: 357-371.

41 Bahi-Buisson N, Bienvenu T: CDKL5-related disorders: from clinical description to molecular genetics. Mol Syndromol 2011; 2: 137-152.

42 Elia M, Falco M, Ferri R et al: CDKL5 mutations in boys with severe encephalopathy and early-onset intractable epilepsy. Neurology 2008; 71: 997-999.

43 Liang JS, Shimojima K, Takayama R et al: CDKL5 alterations lead to early epileptic encephalopathy in both genders. Epilepsia 2011; 52: 1835-1842.

44 Mirzaa GM, Paciorkowski AR, Marsh ED et al: CDKL5 and ARX mutations in males with early-onset epilepsy. Pediatr Neurol 2013; 48: 367-377.

45 Sartori S, Di Rosa G, Polli R et al: A novel CDKL5 mutation in a 47,XXY boy with the early-onset seizure variant of Rett syndrome. Am J Med Genet A 2009; 149A: 232-236.

46 Kalscheuer VM, Tao J, Donnelly A et al: Disruption of the serine/threonine kinase 9 gene causes severe X-linked infantile spasms and mental retardation. Am J Hum Genet 2003; 72: 1401-1411.

47 Montini E, Andolfi G, Caruso A et al: Identification and characterization of a novel serine-threonine kinase gene from the Xp22 region. Genomics 1998; 51: 427-433.

48 Scala E, Ariani F, Mari F et al: CDKL5/STK9 is mutated in Rett syndrome variant with infantile spasms. J Med Genet 2005; 42: 103-107.

49 Seltzer LE, Paciorkowski AR: Genetic disorders associated with postnatal microcephaly. Am J Med Genet C Semin Med Genet 2014; 166C: 140-155.

50 Brunetti-Pierri N, Berg JS, Scaglia F et al: Recurrent reciprocal 1q21.1 deletions and duplications associated with microcephaly or macrocephaly and developmental and behavioral abnormalities. Nat Genet 2008; 40: 1466-1471.

51 Shinawi M, Liu P, Kang SH et al: Recurrent reciprocal 16p11.2 rearrangements associated with global developmental delay, behavioural problems, dysmorphism, epilepsy, and abnormal head size. J Med Genet 2010; 47: 332-341.

52 Crespi B. Stead P, Elliot M: Comparative genomics of autism and schizophrenia. Proc Natl Acad Sci USA 2010; 107(Suppl 1): 1736-1741.

53 Bahi-Buisson N, Kaminska A, Boddaert N et al: The three stages of epilepsy in patients with CDKL5 mutations. Epilepsia 2008; 49: 1027-1037.

Supplementary Information accompanies this paper on European Journal of Human Genetics website (http://www.nature.com/ejhg) 